\title{
Eine verborgene Klammer der schweizerischen Berufsbildungspolitik
}

\section{Urs Kiener}

In diesem Aufsatz wird anhand einer Fallstudie das Policy-Making der schweizerischen Berufsbildungspolitik analysiert. Als Fallstudie dient die Überfïhrung der Gesundheitsberufe in die Bundeskompetenz im Zusammenhang mit dem neuen Berufsbildungsgesetz. In einer diskurstheoretischen Perspektive zeigt sich, dass es beim Fallbeispiel zwar um die konkrete Verschiebung vom einen in den anderen Kompetenz-Bereich und darüberhinaus um die Durchsetzung eines bestimmten Berufsbildungs-Modells geht. Zugleich aber dreht sich das Policy-Making darum, die Berufsbildung als spezifische, sozial ausgehandelte Wirklichkeit zu bestätigen, sie abzugrenzen und ihre Identität zu behaupten.

\section{Einleitung}

Nach wie vor und besonders auch bezüglich der Berufsbildungspolitik ist die Auffassung weit verbreitet, beim Policy-Making gehe es darum, dass in einem definierten Feld definierte Akteure Lösungen für partikulare Probleme finden. Mehr oder wenig unterschwellig wirken dabei spezifische rationalistische Annahmen: etwa jene, dass Ziel-Mittel-Relationen das Verhältnis der Akteure zum Politikfeld dominierten (die Akteure verfolgen ihre Interessen), dass zwischen Subjekten und Objekten politischer Steuerung eine klare Trennung bestehe, oder dass Ziel-Mittel-Relationen auch die Verfahren des Politikprozesses prägten (etwa in der zeitlichen Abfolge und institutionellen Trennung von Problemdefition, Zielformulierung, Implementierung und Evaluation).

Was aber, wenn Steuerungssubjekt und -objekt nur schwer zu unterscheiden sind? In der Berufsbildung beispielsweise spielen die Verbände traditionell eine zentrale Rolle (und zwar in Deutschland und der Schweiz, vgl. Rothe, 2001) und übernehmen parastaatliche Aufgaben. Auch im neuen, auf anfangs 2004 in Kraft getretenen schweizerischen Berufsbildungsgesetz ist von einer «Verbundaufgabe» von Bund, Kantonen und Wirtschaft bzw. Verbänden die Rede, die auf 
einem - bestehenden oder zu schaffenden - Konsens basiere. Was geschieht, wenn diese Konsensbasis schwindet? Das ist, wie die Diskussionen um dieses Gesetz gezeigt haben, zuweilen durchaus der Fall. Können die Verbände umstandslos der Seite der Subjekte oder der Objekte zugeordnet werden?

Was aber, wenn das Politikfeld selbst nicht abgegrenzt ist? Was geschieht, wenn das Politikfeld sich verändert, wenn neue Akteure das Feld betreten, andere bedeutungslos werden? Auch dafür ist das neue schweizerische Berufsbildungsgesetz ein Beispiel: Das Bildungswesen der Schweiz ist Sache der Kantone, sofern nicht ausdrücklich der Bund dafür zuständig ist. Mit der neuen Bundesverfassung, die seit 2000 in Kraft ist, geht die Berufsbildung ganz in die Kompetetenz des Bundes über. ${ }^{1}$ Dies hat insbesondere zur Folge, dass die bislang kantonal geregelten Bereiche der Gesundheit, des Sozialen und der Kunst (GSK) in die Bundeskompetenz überführt werden müssen und damit der gleichen Regelungsinstanz unterstellt werden, die bislang schon für die gewerblich-industriellen und kaufmännischen Berufe zuständig war. Das neue Berufsbildungsgesetz regelt unter anderem diesen neuen Zustand. Es postuliert das Modell der dualen Ausbildung, wie es im gewerblich-industriellen und kaufmännischen Bereich traditionell dominiert, als - variables und flexibles - Einheitsmodell für die ganze Berufsbildung. Die beruflichen Ausbildungen in den Bereichen Gesundheit, Soziales und Kunst aber waren in der Vergangenheit überwiegend nicht dual, sondern schulisch organisiert. Mit anderen Worten: Faktisch erfolgten bisher die beruflichen Ausbildungen in der Schweiz nach zwei unterschiedlichen Modellen (mit klaren Ausprägungen nach Berufsbereichen - und nach Sprachregionen $^{2}$ ) und zwei unterschiedlichen Ordnungen der Regelungskompetenz; als «Berufsbildung» im gängigen Sinn galt jedoch lediglich diejenige des gewerblich-industriellen Typus. Was zunächst als blosse Übertragung der Regelungskompetenz von den Kantonen an den Bund erscheinen mag, erweist sich somit als weit mehr. Aus der Sicht des Bundesamtes für Berufsbildung und Technologie (BBT) handelt es sich um eine "Integration der Berufsbildung", um die Schaffung eines "gemeinsamen Daches», wofür «die gemeinsamen Elemente einer umfassenden Berufsbildung berücksichtigt und systematisiert werden» und eine «einheitliche Sprache» herausgebildet werden soll (EVD, 2003, S. 3). Diese Ausführungen stehen vor dem Hintergrund einer harschen Kritik am BBT, dem zu Beginn der Diskussionen um das neue Berufsbildungsgesetz vorgeworfen worden war, es nehme zu wenig Rücksicht auf Strukturen und Traditionen der neu zu integrierenden Berufs-Bereiche und stülpe ihnen eine wirtschaftsorientierte Sichtweise und Systematik über. In dieser Sicht handelt es sich bei der Übertragung der Regelungskompetenz somit um die Expansion des einen Modells beruflicher Bildung auf Kosten des anderen, das als ebenso bewährt gilt. ${ }^{3}$

Unlängst hat Hajer (2003) darauf hingewiesen, dass der Kontext des Policy-Making sich in jüngster Zeit stark verändert habe. «There are no clear rules and norms according to which politics is to be conducted and policy measures are to 
be agreed upon» (Hajer, 2003, S. 175). Zwar bezieht sich Hajers Argumentation in erster Linie auf transnationale Politikfelder und den Einfluss nicht-staatlicher Akteure wie NGOs, doch gehen seine Überlegungen weit darüber hinaus und sind für die Berufsbildungspolitik durchaus fruchtbar. Denn entscheidend an seiner Argumentation ist das Folgende: Der Kontext des Policy-Making kann nicht (mehr) als stabil vorausgesetzt, sondern muss selbst als diskursiv aufgefasst werden. Unter diesem Kontext ist insbesondere das institutionell-normative Setting des Policy-Making (die Polity) zu verstehen. Lässt man die Voraussetzung der Stabilität fallen, sind prinzipielle Interdependenzen zwischen Policy-Making und ihrem Kontext die Folge. Um solche Interdependenzen geht es bei den eben genannten Beispielen aus dem Feld der Berufsbildungspolitik: Die Struktur der Akteure, die Definition des Politikfeldes, gesetzliche Regelungen, Vorstellungen und Werte - sie liegen weder ausserhalb des Politikproblems noch ausserhalb der Dynamik des Policy-Making. Nur wenn «politico-administrative institutions, societal processes and cultural adherences» (Hajer, 2003, S. 182) ungeprüft als stabil gesetzt werden, kann man Policy-Making allein als Problemlösung auffassen. In der Perspektive Hajers hingegen geht es beim Policy-Making um mehr bzw. um mehreres gleichzeitig: Bei der Aushandlung von Problemlösungen werden gleichzeitig institutionelle Regeln, Normen und Verfahren bekräftigt oder aber verändert. Aushandlungen innerhalb des Spiels verändern potentiell die Regeln des Spiels. Das eine tun heisst zugleich ein anderes tun. ${ }^{4}$ Damit treten neue Fragen ins Blickfeld, beispielsweise: Wie kommt es zu einer bestimmten Problemdefinition? Wieweit schliesst diese Definition bestimmte Akteure und bestimmtes Wissen bereits aus? Auf welche Weise wird Konsens geschaffen, und worauf stützt sich dieser Konsens - auf Überzeugungen, implizite Theorien, empirisches Wissen? Welche Grenzen des Politikfeldes werden durch eine bestimmte Problemlösung gezogen? Wie verbinden sich Durchsetzungsfähigkeit von Akteuren und Problem-Diskurs? Kurz und zusammengefasst stellt diese Perspektive die Frage, worum es bei einer bestimmten Politik geht.

Hier wird also der Vermutung nachgegangen, dass es in der Berufsbildungspolitik um mehr als nur um Problemlösung geht. Zu diesem Zweck wird zuerst der Gegenstand des Policy-Making, die Berufsbildung, kurz charakterisiert (2.). Daran anschliessend wird ein Fallbeispiel präsentiert (3.) und in Anlehnung an diskurstheoretische Ansätze interpretiert (4.), worauf ein Fazit (5.) den Abschluss bildet. 5

\section{Die Berufsbildung - der Gegenstand der Berufs- bildungspolitik}

Systemtheoretisch gesehen ist die Berufsbildung ein Überschneidungsbereich zwischen den zwei Funktionssystemen Wirtschaft und Erziehung (Schriewer, 
1987). Damit sind «organisatorische Arrangements zur Sicherstellung intersystemischer Leistungsbeziehungen auch unter Bedingungen weit getriebener gesellschaftlicher Differenzierung» gemeint: «Institutionalisierte Berufsausbildung stellt vor diesem Hintergrund den anspruchsvoll-riskanten Versuch dar, die Sinn-Orientierungen des Erziehungssystems mit der Rationalität wirtschaftlichen Handelns zu kombinieren» (Schriewer \& Harney, 2000, S. 129). Diese Verschränkungen müssen institutionell-konzeptionelle Ordnungen sowie organisatorische Arrangements/Formen finden, was - und das ist in unserem $\mathrm{Zu}$ sammenhang ein zentraler Punkt - grundsätzlich in unterschiedlicher Weise geschehen kann, wofür also funktionale Äquivalente bestehen. Deren konkrete institutionelle Ausgestaltungen sind "Ausdruck nationaler Sozial- und Kulturgeschichte» (Harney \& Storz, 1994, S. 353). Zumindest im deutschsprachigen Europa nun ist der Beruf Medium bzw. Form, welche Wirtschaft und Erziehung strukturell koppelt und um die herum aus der Berufsbildung ein gesellschaftlicher Funktionszusammenhang geschaffen wurde. Damit ist die «Selbstbezüglichkeit ihrer internen Strukturen und Verarbeitungsmechanismen» (Harney \& Storz, 1994, S. 355) impliziert.

Diese äusserst gedrängte Darstellung kann als Basis dienen für die Diskussion von Entwicklungen und Fragestellungen. Beispielsweise lässt sich das Augenmerk auf das Verhältnis zwischen Berufsbildung und Allgemeinbildung richten. Beide kann man als zwei Funktionszusammenhänge, als zwei Konzepte universaler Bildung verstehen (Harney \& Zymek, 1994), die lange Zeit weitgehend beziehungslos nebeneinander bestanden, mit der Bildungsexpansion aber zunehmend in Konkurrenz zueinander gerieten. Seit einigen Jahren hat diese Konkurrenz die Form eines Kampfes um Marktanteile (Schülerzahlen) in einem stagnierenden oder gar rückläufigen Markt angenommen. Folge sind Expansionsversuche und Verteidigungsdispositionen, Annäherungs- und Profilierungsbewegungen. Erinnert sei hier an Image-Kampagnen, die Forderung "gleich langer Spiesse» in Verteilungskämpfen, die Aufrechnung gesellschaftlichen Nutzens etc. Die Politik im einen Bereich nimmt - explizit oder nicht - immer auch Bezug auf den anderen, womit die Verhältnisse zwischen den Bereichen ständiger Veränderung unterworfen werden. Mit anderen Worten: Die Entwicklung der Berufsbildung wird neuerdings von Faktoren beeinflusst, die bislang für sie ohne Belang waren. Wie und worin sich das konkrete institutionelle Arrangement «Berufsbildung» in diesen Auseinandersetzungen ändert, ist eine bedeutsame Frage - für die Entwicklung der Berufsbildung wie für die Möglichkeiten der Berufsbildungspolitik. Nicht zu übersehen ist im Übrigen, dass sich Berufsbildung und Allgemeinbildung weitgehend geschlossen in dem Sinn verhalten, dass Steuerungsobjekt und politisch-administrative Steuerungssubjekte als ununterscheidbarer Funktionszusammenhang auftreten.

Ein anderes Beispiel: Wenn Berufsbildung als Überschneidungsbereich aufgefasst wird, wenn darin Betrieb und Schulen als Vertreter der Systeme Wirtschaft und Erziehung zusammenkommen und in der Form Beruf eine Vermitt- 
lung ihrer Systemlogiken finden, dann müssten in der Berufsbildung zumindest drei Handlungslogiken zu unterscheiden sein, nämlich die betriebliche, die schulische und die berufliche. Harney und Rahn (2000, S. 773) sprechen deshalb von einer «Pluralität der Handlungslogiken» - und zwar sowohl als "Strukturmerkmal» als auch als "Steuerungserfordernis» des beruflichen Bildungswesens. Sie stellen die Gefahr fest, dass die heutige Berufsbildungspolitik diese Handlungslogiken verdoppelt, statt ihre Aufmerksamkeit auf die Schnittstellen zu konzentrieren und die Koppelung der Handlungslogiken zu bearbeiten.

\section{Ein Fallbeispiel einer politisch-administrativen Reform}

Vor dem Hintergrund der ersten beiden Abschnitte soll nun ein Fallbeispiel dargestellt werden, anhand dessen die Frage diskutiert wird, was verhandelt und entschieden wird, wenn eine Sachfrage verhandelt und entschieden wird. Als Fallbeispiel dient die Überführung der Gesundheitsberufe in die Bundeskompetenz, die bereits im ersten Punkt erwähnt wurde, und zwar eingeschränkt auf die Pflegeberufe.

Reformauftrag: Die Einführung von Fachhochschulen (und des Zugangsausweises Berufsmatur) in den 1990er-Jahren zunächst für die Bereiche Technik und Wirtschaft und die Diskussionen um ein neues Berufsbildungsgesetz gaben schon vor der Verabschiedung entsprechender Gesetze die zwingende Erwartung vor, die auf die Berufsbildung im Gesundheitswesen gerichtet war: die Transition ihrer bestehenden Ausbildungsstruktur in die dominierende Systematik sowie die Übertragung der Regelungskompetenz von den Kantonen an den Bund. Diese Systematik sieht einen beruflichen Erstabschluss (hauptsächlich in der Form des eidgenössischen Fähigkeitszeugnisses) auf der Sekundarstufe II vor, auf den anschliessend - auf der Tertiärstufe - Diplome Höherer Fachschulen bzw. von Fachhochschulen folgen sollen (ausserdem Höhere Berufsprüfungen wie Meisterdiplom etc.). Die bisherige Struktur im Gesundheitsbereich jedoch unterlief die Unterscheidung in Sekundarstufe II und Tertiärstufe. Im Wesentlichen bestand sie aus den beiden folgenden Varianten: Besuch einer allgemeinbildenden Vollzeitschule (Diplommittelschule oder Gymnasium) und anschliessende berufliche Ausbildung in Krankenhäusern und Schulen für Pflegeberufe, oder Sprachaufenthalte und Praktika bzw. Berufslehre in einem anderen Bereich und anschliessend die berufliche Ausbildung. Dabei schliesst die berufliche Ausbildung auf zwei Niveaus nach einer drei- bzw. vierjährigen Ausbildung ab. Im Gesundheits- wie auch im Sozialbereich bestand das überwiegende Selbstverständnis, dass ihre Berufstätigkeiten durch die Arbeit mit Menschen und deren Schicksale eine speziell hohe Verantwortung und menschliche Reife voraussetzten, wozu ein Mindestalter von 18 Jahren zwingend notwendig sei. Die ent- 
scheidenden Fragen, für die in der Reform Antworten gefunden werden müssen, sind deshalb die folgenden: Wie ist es möglich, die bestehenden Ausbildungsstrukturen an die verlangte neue Systematik anzupassen? Ist es möglich bzw. sinnvoll, die vollschulische Vorbildung auf Sekundarstufe II beizubehalten? Ist das Modell der dualen Ausbildung (Berufslehre) auf Sekundarstufe II mit dem genannten Selbstverständnis, dass nämlich für diese Berufe persönliche Reife unverzichtbar sei, vereinbar?

Akteurstruktur: Die Veränderung der Akteurstruktur wäre eine eigene Analyse wert. Interessant ist vor allem die eigentliche Reformphase als Zwischen-Phase des Übergangs, in welcher die institutionellen Regeln vor der Reform nicht mehr, diejenigen nach der Reform aber noch nicht gelten. Selbstverständlich finden darin Aushandlungsprozesse um Einflussbereiche und Zuständigkeiten zwischen den Akteuren statt. Es ist dies eine Form des «institutional void», von dem Hajer (2003) spricht, denn diese Prozesse finden auf transitorischem Terrain statt. Deshalb war die Einigung der hauptsächlichen Akteure auf eine gemeinsame Projektorganisation und auf Leitlinien für die laufenden Arbeiten und Entscheide ein notwendiger Bestandteil der Aushandlungsprozesse. Ihr Gelingen setzt ein Minimum an «cultural adherences» voraus, oder: "politics and policy making thus are not simply about finding solutions for pressing problems, but are as much about finding formats that generate trust among mutually interdependent actors» (Hajer, 2003, S. 184)

Rekonstruktion: Nehmen wir das Resultat vorweg: Am Ende der Reform stehen verbindliche Regelungen über den neu geschaffenen Beruf «Fachangestellte/ $r$ Gesundheit» mit Abschluss auf Sekundarstufe II sowie über zwei Abschlüsse auf der Tertiärstufe: das Berufsdiplom Pflege («dipl. Pflegefachfrau/Pflegefachmann») der Stufe Höhere Fachschule und das Fachhochschul-Diplom. (Die beiden Abschlüsse sollen auch nacheinander innerhalb eines einzigen Ausbildungsgangs an der FH erworben werden können.) Auch wenn die Vorschläge und Diskussionen zuweilen in unterschiedlichen Gremien geführt wurden, nahmen die Aushandlungen über diese drei Abschlüsse selbstverständlich immer eng aufeinander Bezug. Hier konzentrieren wir uns auf den Abschluss auf der Sekundarstufe II.

Im Anschluss an verschiedene Expertenberichte erarbeitet eine vierköpfige «Task Force Berufsbildung im Gesundheitswesen» im Auftrag der Schweizerischen Sanitätsdirektorenkonferenz (SDK) «ein konkretes Modell für die künftige Gestaltung der beruflichen Ausbildung (ohne Fachhochschulausbildung) auf der Sekundarstufe II und der Tertiärstufe» (Marty, Perrinjaquet, Hodel \& Plattner, 1998, S. 11). Die Task Force schlägt ein Modell mit zwei Wegen vor: einen «berufsgestützten» und einen «schulgestützten». Beide Wege bestehen aus differenzierten modulförmigen Ausbildungsteilen mit unterschiedlichen Abschluss- und Berechtigungsausweisen. Das Einführungsjahr im «berufsgestützten» Weg bezieht sich auf Gesundheits- und Sozialberufe. 
Die Vernehmlassung des Task-Force-Berichtes ergibt gewisse Straffungen (Plattner, 1999): Der «berufsgestützte» Weg erfolgt über «eine berufsfeldbezogene 3-jährige Ausbildung zur ,Fachangestellten Gesundheit und Soziales' mit Vertiefungsmöglichkeiten». Im schulischen Weg folgt auf einen anerkannten schulischen Sekundarstufe-II-Abschluss «ein flexibel zu gestaltendes ,Modul zur Schaffung einer einheitlichen Grundlage' (Homogenisierung)», das ebenfalls zum Abschluss «Fachangestellte/r Gesundheit und Soziales» führt.

Im Jahr 2001 treten die Arbeiten, die - nach der grundsätzlichen und formellen Genehmigung der Bildungssystematik durch die SDK 1999 - von verschiedenen Gremien geleistet werden, in ihre entscheidende Phase: Das SDK gibt zusammen mit dem Schweizerischen Roten Kreuz (SRK) - das in Leistungsverträgen von den Kantonen mit der Regelung der Berufsbildung im Gesundheitsbereich betraut war - ein "Berufsprofil Fachangestellte/r Gesundheit» (FAGE) und ein "Berufsdiplom Pflege» - also den Diplomabschluss aufTertiärstufe) in die Vernehmlassung. Das Berufsprofil Fachangestellte/r Gesundheit sieht zwei gleichwertige Ausbildungsformen vor: eine duale Berufslehre mit einem schulischen Unterrichts-Anteil von mind. 30\% und den Besuch der sogenannten Berufsfachschule. In beiden Ausbildungsformen soll die Ausbildung mit einem Basislehrjahr beginnen. Von einem Berufsfeld "Gesundheit und Soziales» ist nicht mehr die Rede. Die Berufsfachschule wurde in die Diskussion des neuen Berufsbildungsgesetzes eingebracht; sie sollte für «kognitiv anspruchsvolle» Bereiche Ausbildungen anbieten, die zu mehr als der Hälfte aus schulischen Anteilen bestehen, zumindest ein Jahr Praktikum enthalten und wie die traditionelle Ausbildung mit dem eidgenössischen Fähigkeitszeugnis abschliessen. - Damit wird ein Kompromiss vorgelegt, der sehr ausgewogen scheint, weil er unterschiedlichste Ansprüche gleichzeitig zu befriedigen vermeint: Die traditionelle Ausbildung über die schulische Sekundarstufe II wird weitgehend beibehalten; damit kommt man den Trägern der bisherigen Ausbildung entgegen. Allerdings wird dafür das Gefäss eines neuen, vom BBT propagierten Schultyps vorgesehen, der insbesondere die Diplommittelschule ablösen soll. Ihr zur Seite gestellt wird eine duale Lehre. Damit trägt man den Ansprüchen und dem Druck der neuen Bildungssystematik und ihrer Vertreter Rechnung. Dabei werden aber - durch schulisches Basisjahr und erhöhten Allgemeinbildungsanteil - die Bedenken bezüglich Alter und persönlicher Reife berücksichtigt.

Überraschend wird in der Vernehmlassung der höhere schulische Anteil deutlich abgelehnt. Und bereits 3 Monate nach der Publikation der Vernehmlassungsauswertung liegt die von SRK und SDK genehmigte "Bildungsverordnung Fachangestellte/r Gesundheit» vor, welche auf den 1. Juli 2002 in Kraft tritt. Dieser Ausbildungsgang ist klar dual organisiert; die Zahl schulischer Lektionen ist in Artikel 14 verbindlich auf 1'800 (das Niveau anderer Berufe) festgesetzt, wobei «die Verteilung auf die Lehrjahre [...] regionale Gegebenheiten berücksichtigen» könne. Mit der Bildungsverordnung sind die jahrelangen Arbeiten von Expertengruppen und Fachorganisationen in einem wesentlichen Punkt abrupt 
zurückgewiesen worden: nämlich im Versuch, möglichst viele Elemente der traditionellen Ausbildung im Gesundheitsbereich in die neue Systematik zu retten. Die Lehre FAGE unterscheidet sich strukturell in nichts von der für andere Berufe dominanten Ausbildungsorganisation. ${ }^{6}$ Interessanterweise fand im gleichen Jahr ein ähnlicher Wechsel in der Diskussion des Berufsbildungsgesetz statt: In der letzten Phase der parlamentarischen Beratung wurde die skizzierte Berufsfachschule entgegen den Vorschlägen von Expertenbericht und Bundesamt nicht aufgenommen und stattdessen der Passus verabschiedet, dass "die Anteile der Bildung, ihre organisatorische Ausgestaltung und die zeitliche Aufteilung [...] nach den Ansprüchen der Berufstätigkeit in der entsprechenden Bildungsverordnung bestimmt» (Art. 16, Absatz 3), also den Aushandlungen auf der Ebene der Branchenverbände überlassen werden. ${ }^{7}$

Wenn wir auf die entscheidenden Reform-Fragen zurückkommen, dann lässt sich aus diesem Resultat des Reformprozesses der Schluss ziehen, dass weit grössere Änderungen und eine weit grössere Abkehr von der bisherigen Ausbildungsstruktur «möglich» war, als in den Diskussionen unter Experten und Steuerungskonferenzen dargestellt wurde. Allerdings: «möglich» in welcher Hinsicht, bezüglicher welcher Referenz? Interessant ist es deshalb, die Argumentationen im Reformprozess zu betrachten.

Argumente und Referenzen im Reformprozess: Die folgenden Resultate basieren auf unterschiedlichen Analyseschritten (Argumentenanalyse, Auszählung von Worthäufigkeiten, inhalt- und kontext-interpretierende Gruppendiskussionen unter Personen mit sehr unterschiedlicher Nähe zum Thema und unterschiedlichem disziplinären Zugang), welchen die ausgewählten Texte in einem iterativen Verfahren unterzogen wurden.

Diese Analysen führen zu Ergebnissen, die auf den ersten Blick erstaunen mögen. Für jene Bereiche der Texte, die sich mit Analysen, Begründungen, Legitimationen etc. beschäftigen, ergibt sich nämlich das Bild einer Ausblendung von empirisch vorfindbarer und zu interpretierender Wirklichkeit durch eine stark nominalistisch geprägte und überwiegend selbstbezügliche Argumentation. Beispiele:

- Die Texte führen keine Auseinandersetzung mit empirisch gestützten Aussagen über die Wirklichkeit des Politikfeldes, sie behaupten wie selbstverständlich Trends und Entwicklungen, ohne Bezug auf (wissenschaftliche) Literatur zu nehmen.

- Die Texte führen keine Auseinandersetzung mit (anderen) Argumenten, sie setzen Aussagen ohne Bezug auf andere.

- Die Texte sind begrifflich-konzeptionell unscharf, die verwendeten Begriffe werden analytisch nicht dimensioniert, sondern ungeklärt nebeneinander und miteinander verwendet (z.B. Beruf, Tätigkeit, Arbeit; Arbeitsfeld, Berufsfeld; Ausbildungsgang, Ausbildungsweg etc.). 
- Akteure, Bezugs- und Anspruchsgruppen werden nur als kategoriale Einheiten eingeführt («der Arbeitsmarkt», "die Jugendlichen», "die Gesellschaft»).

Das Anliegen der begründenden und erläuternden Textteile scheint begriffliche Strukturierung zu sein, und zwar Strukturierung, die es erlaubt, das eine wie das andere anzuerkennen und zu propagieren. Es handelt sich um begriffliche und rhetorische Plattformen des «sowohl-als auch». Sie schliessen nicht aus, setzen kein «entweder-oder», sondern konstruieren Räume, in denen Vereinbarkeiten und Kompromisse immer möglich sind. Dieses Ergebnis lässt selbstverständlich die Frage nach der Funktion dieser Texte aufkommen. Zunächst ist - gestützt auf die Experteninterviews - festzuhalten, dass Texte als Medium dieses politisch-administrativen Aushandlungsprozesses eher die Ausnahme sind: Denn potentiell legen sie fest und machen behaftbar - was in solchen Prozessen oft vermieden werden soll. Die hauptsächliche Funktion von Begründungen und Erläuterungen ist es offenbar, Konsens zu ermöglichen bzw. nominale Anschlussfähigkeit zu schaffen. Ihre Funktion ist es also gerade nicht, Wissen über die zu steuernde Wirklichkeit mit expliziten Politikzielen nachvollziehbar und überzeugend zu kombinieren und der rationalen Diskussion auszusetzen. ${ }^{8}$ Diese Form des Policy-Making gewichtet analytische und konzeptionelle weit geringer als politische Konsens- und Prozessaspekte. Ihre Referenz ist weit weniger die Wirklichkeit des Politikfeldes, indiziert durch empirisch gesättigtes Wissen über das Feld, als vage definierte, vieldeutige Begriffe und eine vertraute Rhetorik. Sie kann es in Kauf nehmen, wenn die Verbindungen zwischen Argumentation und Lösungsvorschlag nur lose oder ungeklärt und wenn die Begründungen selbst nicht stringent sind. Trotz bzw. wegen dieser Beschränkung auf die nominale Ebene: Es werden Entscheide gefällt. ${ }^{9}$

Aus diesen Befunden folgt eine scheinbar paradoxe Form von Aushandlungsund Entscheidungsprozessen: Der Entscheid für eine Lösung, der immer auch andere Lösungen ausschliesst, basiert nicht auf Argumenten, deren Überzeugungskraft diejenige anderer Argumente übertrifft und diese anderen Argumente damit als entscheidungs-relevante ausschliesst. Der Ausschluss von Lösungs-Alternativen geschieht im Gegenteil auf der Basis von nicht-ausschliessenden begrifflichen Strukturierungen des Problems. Das heisst: Der Ausschluss von politischen Entscheidungs-Alternativen basiert - rhetorisch - auf Texten, die manifest gar nichts ausschliessen; Entscheidungen werden scheinbar ohne Entscheidungen gefällt. Das bedeutet: Die Entscheidungen setzen «etwas» voraus und basieren auf «etwas», das in den Texten nicht erscheint. Oder umgekehrt: Die Texte (Begründungen, Erläuterungen, Rechtfertigungen) lassen «etwas» weg, enthalten einen weissen Fleck, eine Leerstelle. 


\section{Eine Interpretation}

Wie funktioniert diese paradoxe Form von Policy-Making, wie ist sie möglich? Zur Beantwortung dieser Frage könnte man der Spur folgen, dass hier eine gegenüber der Politikfeld-Wirklichkeit und gegenüber der politischen Praxis verselbständigte mächtige Rhetorik am Werke sei, eine rein "symbolische» Politik im abwertenden Sinn, welche die «tatsächlichen» Gegebenheiten und Kräfte ausklammert. Nur: Hajers (2003) Hinweis, dass inhaltliche, prozessuale und institutionelle Seiten der Politik nicht apriori zu trennen seien, lässt es ratsam erscheinen, andere Wege einzuschlagen. Eine Möglichkeit dafür sind theoretische Ansätze, welche Sprache als wirklichkeitskonstituierend auffassen; und eine Variante davon sind Diskurstheorien, welche Diskurse zugleich als Praktiken und Wissen verstehen. In einer solchen Perspektive schaffen Diskurse Bedeutung und Sinn, und zwar in und durch Handlungen, diskursive Praktiken. Institutionen bzw. sozial ausgehandelte Wirklichkeit würden demnach zumindest teilweise aus Praktiken bestehen, die auf gemeinsamer Bedeutung basieren, bzw. auf Bedeutungen («cultural adherence»), die mit gemeinsamen Praktiken verbunden sind. So gesehen ist das Politikfeld Berufsbildung nicht einfach «da», unabhängig von Handlungen von Akteuren, sondern es wird permanent durch die in ihr stattfindenden sozialen Praktiken (re-)konstituiert. Und so gesehen kann PolicyMaking als diskursive Praxis gesehen werden, als Schaffung und Aufrechterhaltung von wirklichkeitsordnender Bedeutung (siehe z.B. die Beiträge in Angermüller, Bunzmann \& Nonhoff, 2001; Howarth, Noval \& Savrakakis, 2000; Keller, Hirseland, Schneider \& Viehöver, 2001). Kommen wir auf unsere Analyse von Argumenten und Referenzen zurück und beziehen wir sie erneut auf die Berufsbildung und die Berufsbildungspolitik.

Die Analyse der Fallstudie hat zum Schluss geführt, dass Konflikte ausgetragen und politische Lösungen gefunden werden, aber auf der Grundlage von «etwas», das dabei ausgeklammert und dem Aushandlungsprozess und den Konflikten entzogen wird. In Anlehnung an diskurstheoretische Überlegungen könnte man vermuten, dass hier etwas ausgeklammert wird, um es unantastbar zu machen; und unantastbar soll es sein, weil es die latente Funktion hat, die Gemeinsamkeit, die Kohärenz, die Identität der Institution zu gewährleisten. Wir haben gesehen, dass einerseits nominale Kompromisse vorbereitet und ermöglicht werden, indem Begriffe offen, mehrdeutig und anpassungsfähig gehalten werden, und dass andererseits - davon getrennt, aber auf ihrer Basis - die konkreten Aushandlungen stattfinden. Wie auch immer sie ausgehen - die beteiligten Akteure stehen auf dem gleichen nominalen Boden. Unter dem Dissens liegt der Konsens. Aber die Operation, die Bedeutung von Begriffen variabel zu halten, ist freilich nur dann erfolgversprechend, wenn sie so zusammengehalten werden, dass die «Identität» des Diskurses erhalten bleibt. Notwendig ist also eine nicht-beliebige Klammer: ein gegenüber den anderen Begriffen privilegierter und in privilegierten Praktiken verankerter Bedeutungsträger bzw. entspre- 
chende privilegierte Praktiken. ${ }^{10}$ Zwar geht es beim betrachteten Fallbeispiel von Policy-Making um die Überführung der Ausbildung für die Gesundheitsberufe von der kantonalen in die Bundeskompetenz und darüberhinaus um die Durchsetzung eines bestimmten Berufsbildungs-Modells. Gleichzeitig aber dreht sich das Policy-Making darum, die Berufsbildung als spezifische sozial ausgehandelte Wirklichkeit - «Ausdruck nationaler Sozial- und Kulturgeschichte» zu bestätigen. Diese verborgene Funktion hat im Fall der Berufsbildung - wenn das Fallbeispiel verallgemeinert werden darf - interessante spezifische Aspekte.

Die Abkoppelung der Entscheid-Begründungen von einer empirisch vorfindbaren und zu interpretierenden Wirklichkeit schafft dieser Wirklichkeit ein «Eigenleben» jenseits von Interpretation und Interpretationskonflikten: Sie ist wie sie ist. Gleichzeitig aber wird auch vorausgesetzt, dass ihre Plastizität genügend gross ist, um die getroffenen Lösungen zuzulassen. Sie ist wie sie ist, aber sie wird es wohl auch vertragen, ein bisschen anders zu sein. Es ist das ein Verhältnis, das auf bewusste und geplante Steuerung verzichtet (dafür wäre empirisch gehaltvolles Wissen notwendig) im Vertrauen darauf, dass «es» sich schon richten wird. $\mathrm{Zu}$ diesem Muster passt das in der Berufsbildungspolitik verbreitete Verfahren, sich auf Einheits-Lösungen und die (mehr oder weniger stillschweigende) Möglichkeit differenter, gar disparater, Umsetzung zu einigen. ${ }^{11}$ Diese Wirklichkeit, die ist, wie sie ist, aber auch ein wenig anders sein kann - das ist nicht die äussere Wirklichkeit der üblichen empirisch arbeitenden Sozialforschung oder des idealisierten politischen Akteurs, der seine Interessen rational verfolgt und dafür die Funktionsweise des Politikfeldes möglichst genau kennen will. Diese Wirklichkeit ist aber auch nicht diejenige einer konstruktivistischen Position, welche Wirklichkeit als sozial konstituiert und nicht als etwas objektiv Gegebenes nimmt. Was wir hier antreffen, ist - unbefangen formuliert - die Vorstellung einer völlig unproblematischen Wirklichkeit, eine Wirklichkeit, die deshalb kein Problem ist, weil man selbstverständlicher Teil davon ist und darin unproblematisch handelt. Es ist die Wirklichkeit der sogenannten «Praxis». Ohne hier näher darauf eingehen zu können, soll nur darauf hingewiesen werden, dass «Praxis» in der Berufsbildung ein umfassendes, unspezifisches, multidimensionales, flexibles Konzept ist, dessen Bedeutung je nach Kontext hochgradig variiert - insbesondere je nach der Opposition bzw. dem Andern, gegenüber dem das Konzept eingesetzt wird.

\section{Ein Fazit}

Im Rahmen der skizzierten Fallstudie erscheint Policy-Making sowohl als Problemlösung als auch als Aufrechterhaltung des institutionellen und kulturellen Rahmens der Berufsbildung bzw. Berufsbildungspolitik. Folgt man der verallgemeinerten These, dass das Konzept «Praxis» (oder das Diskurselement «Praxis») in der Berufsbildung eine privilegierte wirklichkeitskonstituierende Rolle 
spielt, dann ergeben sich einige interessante Folgen: Die system- und steuerungstheoretisch identifizierten Problemlagen der Berufsbildung werden nicht systematisch, sondern "von Fall zu Fall» bearbeitet. Was theoretisch-analytisch differenziert wurde, bleibt in der spezifischen Wirklichkeits- und Praxis-Vorstellung vermischt. Das betrifft nicht nur die Unterscheidung von Berufsbildung und Berufsbildungspolitik, sondern es betrifft auch die inhärenten Konflikte des Überschneidungsbereichs Berufsbildung und die unterschiedlichen Funktionslogiken, die in ihm wirksam sind. Im skizzierten Fallsbeispiel werden diese Logiken addiert - was ihre Spannungen gerade nicht aufhebt. Und vor allem wird die Unterscheidung zwischen Betrieb und Beruf nicht verfolgt - sie scheint im Konzept «Praxis» aufzugehen (vgl. zu dieser Grenzverwischung detailliert Schriewer \& Harney, 2000). Genau damit aber bearbeitet die Berufsbildungspolitik die Grenze zum Funktionszusammenhang Allgemeinbildung - dem Konkurrenten um Schülerströme und dem Aussen, demgegenüber sich die Berufsbildung abgrenzt und ihre Identität aufrecht erhält: Die schulische Logik bzw. die Logik des Bildungssystems (die stark auf Berechtigungen ausgerichtet ist) bedroht die Berufsbildung zunehmend von aussen wie von innen. Ihr wird nun offenbar von der Berufsbildung das Konzept «Praxis» entgegengesetzt - als hauptsächliches Unterscheidungsmerkmal und als hauptsächliches Element der Identitätsbehauptung. Es wäre zu untersuchen, welche Folgen diese Verwischung der Differenz zwischen Beruf und Betrieb für die Berufsbildung und Berufsbildungspolitik hat (vgl. Kiener, 2004). Das sollte mit Vorteil in der Perspektive geschehen, die spezifische Rationalität des Policy-Making in der Berufsbildung herausarbeiten und dabei nicht nur deren Nachteile, sondern auch deren Vorteile spezifiziert in den Blick nehmen zu wollen.

\section{Anmerkungen}

1 «Der Bund regelt die Berufsbildung» (Art. 63, Absatz 1).

2 Vollzeit-Berufsschulen nach der Definition des Bundesamtes für Statistik (z.B. Handelsmittelschulen, Lehrwerkstätten, Krankenpflegeschulen etc.) sind in der Suisse romande weit häufiger als in der Deutschschweiz verbreitet. Zusätzlich ist der Anteil von allgemein bildenden Vollzeitschulen auf der Sekundarstufe II in der Suisse romande wesentlich höher als in der Deutschschweiz - entsprechend geringer der Anteil der Berufsbildung.

3 Selbstverständlich sind diese Hinweise selektiv; sie bezwecken lediglich die Gegenüberstellung von Äusserungen, die zu einem bestimmten Zeitpunkt relevant waren, ohne auf deren Hintergrund einzugehen.

4 Diese Perspektive der «Nicht-Trennung» ist selbstverständlich keineswegs neu, sondern steht in einer langen sozialwissenschaftlichen Tradition, die auf den Pragmatismus zurückgeht und heute als Teil der als post-positivistisch, konstruktivistisch u.a. bezeichneten sozialwissenschaftlichen Richtungen gilt.

5 Die folgenden Überlegungen sind zu einem Grossteil im Rahmen eines Projektes im NFP 43 des Schweizerischen Nationalfonds (Projektnummer 4043-58351) entwickelt worden. Darin wurden u.a. mehrere Fallstudien durchgeführt; eine davon ist die Grundlage für das hier präsentierte Fallbeispiel. Sie stützt sich auf Experteninterviews und auf die Auswertung öffentlich zugänglicher und von den Experten zur Verfügung gestellter Texte. Ich 
danke Jolanda Blum und Michael Schanne, die beide am Projekt mitarbeiteten, für ihre hilfreichen Kommentare zu diesem Text. Weiter habe ich zwei anonymen Gutachtern für ihre Kommentare zu danken.

6 Es soll ausdrücklich betont werden, dass eine Bewertung dieses Reformresultates hier nicht zur Debatte steht, genausowenig eine Bewertung der inhaltlichen Positionen und Argumente der Akteure.

7 Dass einige Monate später, im Jahr 2003, die Existenz der gefährdeten Diplommittelschule als Fachmittelschule in kantonaler Kompetenz im Wesentlichen bestätigt und damit ihre Rolle im schulischen Weg bekräftigt wurde, ist gewiss auch mit der Ablehnung der Berufsfachschule in Verbindung zu bringen.

8 Hier wären nun selbstverständlich Differenzierungen der Funktion von Texten nach Phasen des Politikprozesses und Textsorten notwendig. Der Hinweis muss hier genügen, dass das genannte Resultat sehr wohl auch für den Expertenbericht gilt.

9 Es liesse sich einwenden, mit den Vernehmlassungsantworten breche die ausgeschlossene Wirklichkeit in die Reformdiskussion ein. Nur: Von den Vernehmlassern des Jahres 2001 (Kantone, Verbände etc.), die in ihrer Mehrheit beim Ausbildungsgang für eine Reduktion der Lektionenzahl von 2'800 auf 1'800 plädierten und auch andere detaillierte Fragen präzis zu beantworten wussten, gibt die Mehrheit an, dass sie keine Angaben zum zukünftigen Bedarf an Fachangestellten Gesundheit machen könnten (De Kaenel et al., 2002, S. 30).

10 Wir beziehen uns hier auf Laclau und Mouffe und deren Diskussion leerer und privilegierter Signifikanten; vgl. neben der genannten Literatur auch Stäheli (1999).

11 Ein Beispiel dafür ist Artikel 16 des neuen Berufsbildungsgesetzes, s. Punkt «Rekonstruktion» im Abschnitt «Ein Fallbeispiel einer politisch-administrativen Reform». Ein anderes Beispiel ist die Einführung der technischen Berufs-matur, wo die "Vollzugspolitik als Auseinandersetzung um Wirklichkeiten» sich allerdings als Steuerungsproblem ausgewirkt hat (vgl. Kiener \& Gonon, 1998, S. 132ff).

\section{Literaturverzeichnis}

Angermüller, J., Bunzmann, K. \& Nonhoff, M. (Hrsg.) (2001). Diskursanalyse. Theorien, Methoden, Anwendungen. Hamburg: Argument.

De Kaenel, M., Mäder, M., Plattner, J.-M., Vollenweider, A.-C., Wohlfender D. \& Zosso, B. (2002). Auswertung der Vernehmlassung zum Fähigkeitszeugnis "Fachangestelle Gesundheit» und zum "Berufsdiplom Pflege». Bern: SDK/SRK.

EVD (2003). Revision der Berufsbildungsverordnung. Erläuternder Bericht für die Vernehmlassung. Bern: EVD.

Hajer, M. (2003). Policy Without Polity? Policy Analysis and the Institutional Void. Policy Sciences, 36, 175-195.

Harney, K. \& Rahn, S. (2000). Steuerungsprobleme im beruflichen Bildungswesen - Grenzen der Schulpolitik. Zeitschrift für Pädagogik, 46, 731-751.

Harney K. \& Storz, P. (1994). Strukturwandel beruflicher Bildung. In D.K. Müller (Hrsg.), Pädagogik, Erziehungswissenschaft, Bildung: eine Einführung in das Studium (S. 353-381). Köln/Weimar/Wien: Böhlau.

Harney, K. \& Zymek, B. (1994). Allgemeinbildung und Berufsbildung. Zwei konkurrierende Konzepte der Systembildung in der deutschen Bildungsgeschichte und ihre aktuelle Krise. Zeitschrift für Pädagogik, 40, 405-422.

Howarth, D., Noval, A.J. \& Savrakakis, Y. (Hrsg.) (2000). Discourse Theory and Political Analysis. Manchester/New York: Manchester University Press.

Keller, R., Hirseland, A., Schneider, W. \& Viehöver, W. (Hrsg.) (2001). Handbuch Sozialwissenschaftliche Diskursanalyse. Band 1: Theorien und Methoden. Opladen: Leske+Budrich. 
Kiener, U. (2004). Gleich und anders. Die Entwicklung der Berufsbildung aus der Perspektive aktueller Reformen. Bern/Aarau: NFP 43 (Synthesis 11).

Kiener, U. \& Gonon, Ph. (1998). Die Berufsmatur. Ein Fallbeispiel schweizerischer Berufsbildungspolitik. Chur/Zürich: Rüegger.

Marty, R., Perrinjaquet, I., Hodel, H. \& Plattner, J.-M. (1998). Bericht der Task Force Berufsbildung im Gesundheitswesen. Bern: SDK.

Plattner, J.-M. (1999). Erläuterungen zum Bericht TASK FORCE. Bern: SDK.

Rothe, G. (2001). Die Systeme beruflicher Qualifizierung Deutschlands, Österreichs und der Schweiz im Vergleich. Wien/Luzern/Villingen-Schwenningen: öbv\&hpt/DBK/neckar-Verlag

Schriewer, J. (1987). Funktionssymbiosen von Überschneidungsbereichen: Systemtheoretische Konstrukte in vergleichender Erziehungsforschung. In J. Oelkers \& H.-E. Tenorth (Hrsg.), Pädagogik, Erziehungswissenschaft und Systemtheorie (S. 76-101). Weinheim/Basel: Beltz.

Schriewer, J. \& Harney, K. (2000). Beruflichkeit versus culture technique: Zu einer Soziogenese arbeitsbezogener Semantik. In P. Wagner, C. Didry \& B. Zimmermann (Hrsg.), Arbeit und Nationalstaat. Frankreich und Deutschland in europäischer Perspektive (S. 128168). Franfurt/New York: Campus.

Stäheli, U. (1999). Die politische Theorie der Hegemonie: Ernesto Laclau und Chantal Mouffe. In A. Brodocz \& G.S. Schaal (Hrsg.), Politische Theorien der Gegenwart (S. 143166). Opladen: Leske+Budrich.

Schlagworte: Bildungspolitik, Berufsbildung, Gesundheitsberufe, Diskursanalyse

\section{Une parenthèse cachée de la politique suisse en matière de formation professionnelle.}

\section{Résumé}

Dans ce texte est analysée, au travers d'une étude de cas, la Policy-Making de la politique suisse en matière de formation professionnelle. L'étude de cas se penche sur les formations de la santé dans la compétence de la Confédération, en lien avec la nouvelle loi sur la profession professionnelle. Dans une perspective théorique et discursive il ressort qu'il s'agit, dans cet exemple, d'un report de compétence d'un champ à un autre et de l'imposition d'un modèle de formation professionnelle bien précis. Parallèlement il est montré que, dans la Policy-Making, il s'agit de confirmer la formation professionnelle en tant que réalité spécifique, sociale et négociée, de la délimiter et d'affirmer son identité.

Mots clefs: Politique de la formation, formation professionnelle, professions de la santé, analyse du discours 


\section{Una parentesi nascosta della politica svizzera della formazione professionale}

\section{Riassunto}

La discussione di un caso costituisce la premessa per analizzare il «policy-making» della politica svizzera della formazione professionale. Il caso è dato dal passaggio, avvenuto a seguito della nuova legge sulla formazione professionale, delle professioni sanitarie dall'ambito di competenza dei Cantoni a quello della Confederazione. Se nel quadro di un discorso teorico, appare come il caso metta in evidenzia il passaggio di competenza e la realizzazione di un nuovo modello formativo, appare pure come la politica messa in atto miri a radicare la formazione professionale sul piano sociale e a fornirle una propria identità.

Parole chiave: Politica formativa, formazione professionale, professioni sanitarie, analisi discorsiva

\section{A hidden bracket in the Swiss vocational education Policy}

\section{Summary}

In this article Swiss policy concerning vocational education will be analysed by means of a case study. The case study used was that of the transfer of the responsibility for medical careers from the medical association to the federal level. Here the new federal law concerning vocational education was also considered. From a discourse theoretical perspective it is shown that the case study deals with the concrete displacement from one competence area to another. It further deals with the planning of a certain vocational training model. At the same time policy making turns around and confirms vocational training to be a specific social negotiated reality, to confine it and create its own identity.

Key words: educational policy, vocational training, medical careers, discourse analysis 
T h e m a 